Research Article

\title{
Bidder Network Community Division and Collusion Suspicion Analysis in Chinese Construction Projects
}

\author{
Jiwei Zhu $\mathbb{D}^{1,2,3}$ Bing Wang $\mathbb{D}^{1,2,3}$ Liang $L i \mathbb{D}^{2}$, and Jiangrui Wang $\mathbb{D}^{1,2,3}$ \\ ${ }^{1}$ State Key Laboratory of Eco-Hydraulics in Northwest Arid Region, Xi'an University of Technology, Xi'an 710048, China \\ ${ }^{2}$ Research Center of Eco-Hydraulics and Sustainable Development, The New Style Think Tank of Shaanxi Universities, \\ Xi'an 710048, China \\ ${ }^{3}$ Department of Engineering Management, Xi'an University of Technology, Xi'an 710048, China
}

Correspondence should be addressed to Jiwei Zhu; xautzhu@163.com

Received 9 October 2020; Revised 16 November 2020; Accepted 5 December 2020; Published 16 December 2020

Academic Editor: Chiara Bedon

Copyright ( 2020 Jiwei Zhu et al. This is an open access article distributed under the Creative Commons Attribution License, which permits unrestricted use, distribution, and reproduction in any medium, provided the original work is properly cited.

\begin{abstract}
Bidder collusion seriously undermines the fair competition of the construction project market, and effective identification of collusion behaviors is of vital importance to the implementation of proactive regulation and supervision. In this paper, the data of construction project bidders from 2011 to 2018 are selected in Shaanxi Province, China, and a bidder network of construction projects is constructed. The collusion suspicion of bidders is analyzed from the macro-, meso-, and microlevels. The results show that the bidder network has features as small world at macrolevels, and it is easy for bidders to involve in collusion. The network community formed by construction, supervision, and survey and design bidding enterprises is analyzed at the mesolevel, and the collusion of supervision enterprises is found to have the highest suspicion At the microlevel, the characteristic value judgment and community division are adopted to analyze the collusion suspicion, which is divided into high, medium, and low according to the possibility. Through a comparison with the actual data, it is found that the method proposed in this paper can effectively identify the collusion behavior of construction project bidders. This paper proposes red, yellow, and green warning mechanism and formulates hierarchical accurate management preparedness, which can provide some suggestions to help prevent bidders from colluding.
\end{abstract}

\section{Introduction}

Bidding has increasingly become an important way to promote free competition in the construction industry market. Bid collusion problems are common in all countries. For example, the United States [1], the Netherlands [2], Japan [3], Italy [4], South Africa [5], and other countries have been battling with and suffering from corruption. Governments of all countries attach great importance to the problem of collusion and adopt a series of policies to prevent collusion (e.g., National Research Council 2011, European Commission 2013, and Australian Government Competition Policy Review 2015) [6]. Not only the national government, other stakeholders have also taken actions to manage and regulate bidding behavior. The Construction Industry Development Board (CIDB), the Organization of Economic Co-operation and Development (OECD) developed several guideline, the Competition Commission of South Africa (CCSA), and the World Bank, which provide the best practice standards and codes of conduct for national and international bidding [7]. Furthermore, to improve the transparency of supervision can be another effective measure to reduce corruption. Although these measures can prevent collusion to a certain extent, collusion is still happening. In addition, long-term collusive bidding has created cartels of construction contractors, and if ignored or undetected for a long time, it will help to establish increasingly organized communities among the winning bidders [8]. Therefore, it is 
of great significance to identify colluding groups in tens of thousands of companies based on bidding behaviors to achieve prior supervision.

Compared with developed countries, developing countries are in a period of focusing on rapid economic development and their bidding system needs to be improved. Therefore, the problem of collusion is more serious. As the largest developing country, with its rapid economic development, its construction industry is also developing rapidly. The construction industry occupies an important position in Chinese national economy [8]. In 2018, the total output value of China's construction industry reached 6.18 trillion yuan, accounting for $6.87 \%$ of the gross domestic product (GDP) [9]. In the past ten years, the bidding area of the total housing construction area has been more than $70 \%$, and in 2018, there were a total of 7,117 engineering bidding agencies in China, and the bidding agency won the bid amount of 156.351 billion yuan, an increase of $14.02 \%$ over 2017 [10], which shows that the position of the bidding system in the construction industry is still very important. However, the "Law of the People's Republic of China on Tendering and Bidding" has only been implemented for 20 years, and there is still a certain gap compared with developed countries. There are 22 specific collusive practices in Chinese construction projects, such as collusive tendering by helping one another [11], which seriously undermines the fair competition in the market [12] and hinders the effective allocation of market resources.

In the bidding activities of construction projects, the collusion between bidders through sharing information and maximizing profits has attracted people's attention [13]. However, such behavior is highly secretive and difficult to supervise, and industry regulators can only investigate if they have sufficient evidence after collusion occurs. As a result, irreparable losses have been caused, and the fair environment of the bidding market has been severely affected. Therefore, effective identification of the construction project bidding collusion suspicion is of great importance to the implementation of proactive regulation and supervision, which helps improve the regulators' ability to cope with the risks caused by collusion.

Collusion has aroused general concern in the fields of economics and sociology. The earliest research on collusion behavior may date back to the concept of tacit collusion in public management, which explains price collusion among enterprises from a static point of view [14]. Laffont and Maskin did preliminary research on the collusion [15]. With continuous development of game theory in bidding research, its application in the analysis of collusion behavior has been extended from the static framework to the dynamic framework [16]. Based on the game theory, Kreps et al. proposed the possibility of group collusion in repeated "prisoner's dilemma" game [17]. Laffont and Martimort applied incomplete contract theory and game theory to the study of collusion behavior in industrial organizations [18]. Many research studies on collusion behavior based on game theory have laid the foundation of game theory in the study of collusion theory. At the same time, the research object of collusion mainly focuses on cartels, such as German cement industry cartel [19], influenza vaccine industry cartel [20], and auction industry cartel [21].

In the study of influencing factors of bidding collusion behavior, Björkman et al. found that closed information auction was more likely to cause collusion than open information auction [22-24]. Padhi and Mohapatra used statistical analysis tools to find that the mean, median, and variance of bid-reserve price ratio of collusion bidders were higher than those of normal bidders, which were used as the basis for testing collusion behavior [25]. Maximereeves studied the evolution of bidder collusion networks suspected of corruption, bid collusion, and bribery based on the analysis of core-edge social networks, finding that collusion bidders are long-term and core participants [26]. Bing et al. believe that the relationship between bidders and officials plays a crucial role in bidding collusion. The establishment of a corrupt relationship enables tenderee to manipulate bidding activities with their power, thus causing collusion [27-30].

In summary, the current relevant research mainly examines the collusion behavior of bidders from the perspective of collusion behavior analysis and its influencing factors. It not only has a solid theoretical foundation but also has some shortcomings. (1) It focuses on the prevention measures of collusion with less concentration on the characteristics of collusion among bidders. (2) The research mostly adopts dynamic game theory and information theory, which cannot be used to study the bidders' participation in collusion at the mesolevel or analyze the behavior of bidders' participation in collusion at the microlevel. (3) Relevant studies are mainly analysis of the collusion behavior after it occurs based on historical data, which have little significance for the supervision before it occurs.

Complex network analysis provides a solution to the collusion problem in construction project bidding. A complex network refers to one with some or all of the properties of self-organization, self-similarity, attractor, small world, and scale free [31-33], which is composed of nodes and edges. Nodes are used to show different individuals, while edges indicate the relationship between individuals. Complex networks based on massive data relationships can be used to analyze data node behaviors at macro-, meso-, and microlevels, which are widely applied in price competition [34], power networks [35, 36], social relationship networks $[32,37]$, and project management [38]. They provided new perspectives for constructing the bidder network of construction projects.

Construction project bidders are connected to form a network under the rules of joint participation, and a complex system is established through constant gaming. Thus, the bidders have a complex relationship in the bidding market [39], which constitutes a complex network with obvious network characteristics. It is necessary to build a complex bidder network for construction projects, study the law of bidder collusion at macro- and mesolevels, analyze the collusion suspicion among bidders at the microlevel, and issue a warning before a collusion behavior occurs.

This paper intends to construct a bidder network and analyze the bidders' collusion suspicion through the network 
characteristic values. The author will construct a bidder network and conduct community division, analyze the bidder community behaviors at the macro- and micro-levels, and compare the differences of possible collusion of different types of bidders. The collusion suspicion of bidders will be analyzed through the network characteristic values, and the collusion bidders are compared with those announced by the regulators to verify the applicability, feasibility, and accuracy of the collusion behavior analysis method. Finally, some industry supervision countermeasures are proposed based on the research findings.

\section{Mathematical Models and Solutions}

2.1. Building a Complex Network of Bidders. Assume that a total of $N$ construction enterprises participate in the bidding. First, a bidder adjacency matrix $\mathrm{A}=\left(a_{i j}\right)_{N \times N}$ is established to reflect the intensity of the bidder's multiple participation in the same project bidding, and based on the adjacency matrix, the bidder's undirected weighted network $G=(V, E)$ is constructed. The bidder network can clearly represent the network relationship between bidders.

2.2. Bidder Network Community Division. The community is a subgroup formed by individuals with a particularly close relationship in the network. The nodes in the community are relatively closely connected, and the connections between communities are relatively sparse [40-42]. Each network can be divided into multiple communities. This paper uses a fast modularity optimization method referred to as BGLL and Cluster Percolation Method (CPM) for community division.

2.3. Division Based on the BGLL. The BGLL algorithm is a cohesion algorithm proposed by Blondel et al. in 2008, based on the concept of modularity, and it is a cohesive algorithm that can be used in the analysis of the hierarchical community structure of the weighted network [43]. The BGLL algorithm includes the two steps of network compression and community reorganization.

Step 1: each node is assigned to a community in the initial network. The number of initial communities is equal to the number of nodes, and then move any node $i$ to the community where its neighbor node $j$ is located, and calculate the change in the $Q$ value of the network. If $\Delta Q$ is greater than 0 , then move the node $i$ to the community with the largest $\Delta Q$. Otherwise, the node $i$ stays in the original community. All nodes go through the above process. The first step stops until that no node moves and the modularity reaches the maximum locally.

Step 2: we build a new network whose nodes are the communities divided in the first step. The edge weight of the new nodes is the sum of the edge weights between the communities. Then, we repeat the first step in the new network to find the maximum modularity. The algorithm stops until the maximum modularity is obtained [44].
The calculation of $Q$ and $\Delta Q$ is as follows:

$$
Q=\frac{1}{2 m} \times \sum_{i j}\left[A_{i j}-\frac{k_{i} * k_{j}}{2 m}\right] \delta\left(C_{i}, C_{j}\right) .
$$

Suppose $A_{i j}$ is the adjacency matrix of the bidder's network, $m$ represents the sum of the edges, and $k_{i}$ and $k_{j}$ represent the degree of nodes $i$ and $j$, respectively. If $i$ and $j$ are divided into the same community, then $\delta\left(C_{i}, C_{j}\right)=1$, and otherwise it is 0 :

$$
\begin{aligned}
\Delta Q= & {\left[\frac{\sum_{\text {in }}+k_{i, \text { in }}}{2 m}-\left(\frac{\sum_{\text {tot }}+k_{i}}{2 m}\right)^{2}\right] } \\
& -\left[\frac{\sum_{\text {in }}}{2 m}-\left(\frac{\sum_{\text {tot }}}{2 m}\right)^{2}-\left(\frac{k_{i}}{2 m}\right)^{2}\right],
\end{aligned}
$$

where $\sum_{\text {in }}$ and $\sum_{\text {out }}$ are the sum of the weights of all edges in community $C$ and edges connecting to the nodes in community $C$, respectively, $k_{i}$ is the sum of the weights of all edges connected to node $I, k_{i, \text { in }}$ is the sum of the weights of the edges that node $i$ connected to nodes in community $C$, and $m$ is the sum of the weights of all the edges in the network.

The maximum value of $Q$ is 1 ; the larger the value of $Q$, the more significant the structure of the network community is. In a real network, the $Q$ value is generally between $[0.3,0.7]$; when $Q$ is greater than or equal to 0.3 , the network has a strong community structure; otherwise, the community structure is not significant [44]. The bidder network community division by the BGLL can identify the bidders' gathering behavior.

2.4. Community Division Based on the CPM. The BGLL divides the network into separate communities, but cannot classify communities that overlap. Therefore, the CPM faction filtering algorithm is used to detect network overlapping and further divide communities. For a given value of $k$, use the overlapping matrix to divide any $k$-factional community and set the elements in the faction matrix with diagonal elements smaller than $k$ and the nondiagonal elements smaller than $k-1$ to be 0 , and the remaining position elements are set to be 1 . An adjacency matrix of the $k$ factional community structure is obtained, and the connected part of the adjacency matrix represents the divided $k$ factional community. In general, the value of $k$ in the CPM faction filtering algorithm is $[4,6][45]$.

\subsection{Community Structure Division Evaluation.}

"Strong/Weak" community is used to evaluate the rationality of community structure division, and the division method is as follows:

$$
\begin{cases}\forall v_{i} \text { fit } S_{i}^{\text {in }}>S_{i}^{\text {out }}, & \text { strong community, } \\ \left(\sum_{i} S_{i}^{\text {in }}\right)>\left(\sum_{i} S_{i}^{\text {out }}\right), & \text { weak community, }\end{cases}
$$

where $s_{i}^{\text {in }}$ represents the internal strength of the bidder $i$, that is, the number of edges between bidder $i$ and other bidders 
within a community and $s_{i}^{\text {out }}$ represents the external strength of the bidder $i$, that is, the number of edges between bidder $i$ and other bidders outside the community.

If only one community in a network-divided community meets the strong/weak community indicators, it means that the community structure of the network is not obvious, that is, the community division is unreasonable, and otherwise, the division of associations is reasonable. Therefore, it can be judged that the strong community is more structural, and the number of bids between the internal bidders and the allied groups is far more than that of the outside members of the alliance, so there is greater suspicion of collusion in bidding.

2.6. Criteria for Suspicion of Collusion. This paper constructed an undirected weighted network of bidders, took the degree, weighted degree of nodes as important indicators in describing the bidder network, and judged the smallworld characteristics of the bidder network based on the degree and weighted degree, centrality index, and clustering coefficient. A bidder network has small-world characteristics, and the contact between bidders is closer [46].

Therefore, we deduced that the possibility of collusion can be inferred from the connection between bidders in the network. The closer the bidders' connection is, the more likely it is to participate in collusion.

2.7. Based on Degree and Weighted Degree. Degree refers to the degree of bidders' participation in joint bidding, while weighted degree indicates the frequency of bidders' participation in joint bidding. Normally, the bidder's own ability is limited, and the number of times participating in bidding within a certain period should be at the average. According to this, it can be determined that the higher the bidder's degree and weighted degree, the more they exceed the average value and the closer their connection. We deduced that the more likely they are, the more will be collusion in bidding.

2.8. Based on the Centrality Index. Centrality index indicates the degree to which a node is at the core of the network. Point centrality, closeness centrality, betweenness centrality, and eigenvector centrality are selected as the centrality indexes of the bidder network to measure the importance of bidders in the network.

In the undirected weighted bidder network, point centrality is used to measure the importance of nodes. The greater the point centrality of a node is, the more important it is in the network. The eigenvector centrality of a node is proportional to the sum of the centrality of its neighboring nodes. The larger the eigenvector centrality is, the more important it is in the network. Closeness centrality reflects the proximity of a node to other nodes. The greater the closeness centrality, the shorter the contact distance with other bidders is. The betweenness centrality refers to the number of shortest paths that the node appears between other nodes. The greater the betweenness centrality is, the more convenient it is to contact other bidders. Therefore, it can be concluded that the greater the centrality of a bidder is, the more important the bidder is in the network, and the easier it is to contact other bidders, and the closer the relationship between them, the greater the suspicion of collusion.

2.9. Based on the Clustering Coefficient. The weighted network clustering coefficient takes into account the edge weight between any bidder and the bidder, and its calculation is as

$$
C_{i}=\frac{\sum_{j, k} w_{i j} w_{j k} w_{k i}}{\left(\sum_{j} w_{i j}\right)^{2}-\sum_{j} w_{i j}^{2}},
$$

where $w_{i j}, w_{j k}$, and $w_{k i}$ represent the weights of the edges between nodes $v_{i}, v_{j}$, and $v_{k}$, respectively.

The clustering coefficient is used to measure the degree to which a bidder and neighbouring bidders participate in bidding together. The average of all bidders' clustering coefficients is the clustering coefficient of the bidder network. Thus, it can be determined that the larger it is, the stronger the connection strength between bidders is, that is, the closer the relationship between bidders is, the greater the possibility of collusion is.

\section{Results and Discussion}

3.1. Bidder Network Construction. The data comes from the Shaanxi Provincial Construction Project Bidding Management Information Network and the Shaanxi Public Resource Trading Center, including the announcements of successful bidding of 7582 construction projects from 2011 to 2018. A total of 5293 bidders were divided into construction, supervision, and survey and design enterprises. (1) The bidders were numbered from 1 to 5293. We used Python to extract the original data and generated the bidders' adjacency matrix. The nodes of the network represent the bidders, and the edges of the network indicate that two bidders participate in the bidding together. The number of bidding by the two bidders determines the weight of the edge and constructs an undirected weighted network. (2) Python was used to calculate the basic properties of the network, and we constructed the bidder network. The number of network nodes is 5293, the number of edges is 19875, and the network density is 0.001 . Therefore, the bidder network was relatively sparse.

3.2. Analysis of Bidder Network Eigenvalues. Through macrolevel analysis, it is found that the bidder network has features as small world. Using Gephi to calculate the eigenvalues (see Table 1), we find the bidder network has a higher average path length of 4.835 . It indicates that any two of the 5,293 bidders can be connected through 4.835 bidders only. The average clustering coefficient of the bidder network is 0.676 , larger than that of the random network of the same scale, which indicates that the network is highly concentrated. Thus, the bidder network has features as small 
TABLE 1: Small-world eigenvalues of the network.

\begin{tabular}{lccccccc}
\hline Node & Edge & $\begin{array}{c}\text { Average } \\
\text { degree }\end{array}$ & $\begin{array}{c}\text { Average weighted } \\
\text { degree }\end{array}$ & $\begin{array}{c}\text { Average path } \\
\text { length }\end{array}$ & $\begin{array}{c}\text { Average clustering } \\
\text { coefficient }\end{array}$ & $\begin{array}{c}\text { Network } \\
\text { diameter }\end{array}$ & $\begin{array}{c}\text { Network } \\
\text { density }\end{array}$ \\
\hline 5293 & 19875 & 7.51 & 12.205 & 4.835 & 0.676 & 17 & 0.001 \\
\hline
\end{tabular}

world at the macrolevel, and the bidders are prone to collusion.

\subsection{Bidder Network Community Division and Collusion} Suspicion Analysis. By classifying the services provided by the nodes included in each community, it is found that the enterprise types include construction enterprise, supervision enterprise, and survey and design enterprise. Therefore, the bidder network is divided into the above three types for analysis at meso level. The basic characteristic values of the three types of enterprise bidder network are shown in Table 2.

The bidder network of the construction enterprise contains 3150 nodes and 41302 edges. The maximum degree of nodes is 155 , the average degree is 9.081 , the maximum weighted degree is 403 , and the average weighted degree is 14.326. The degree distribution of nodes is shown in Figure 1(a), and the weighted degree is shown in Figure 2(a). More than $80 \%$ of construction enterprises' node degree and weighted degrees is less than or equal to average, which is at a normal level, $0.3 \%$ of nodes have a degree of over 100 , and $2.8 \%$ of nodes have a weighted degree of over 100 , or even over 400 , indicating that these nodes are significantly more active in bidding in the market than the average, with certain abnormalities.

There are 412 nodes and 2396 edges in the bidder network of supervision enterprises. The maximum degree of nodes is 84 , the average degree is 11.631 , the maximum weighted degree is 360 , and the average weighted degree is 26.306. The degree distribution of nodes is shown in Figure 1(b), and the weighted degree is shown in Figure 2(b). The weighted degree of $7.3 \%$ nodes is more than 100 . The average degree and weighted degree of supervision enterprises are higher than the average level of all bidders' networks. Compared with construction enterprises and survey and design enterprises, the average degree and weighted degree of supervision enterprises are the largest, which indicates that supervision enterprises participate in bidding activities most frequently within the scope of this paper.

The survey and design enterprise bidder network contain 450 nodes and 1034 edges. The maximum degree of nodes is 66 , the average degree is 4.596 , the maximum weighted degree is 174 , and the average weighted degree is 7.089 . The degree distribution of nodes is shown in Figure 1(c), the weighted degree is shown in Figure 2(c). The degree and weighted degree of more than $92 \%$ of the nodes is less than 20 . The average degree and weighted degree of the survey and design bidder network are significantly lower than the overall level of all bidder networks. Among the three types of enterprises, the average degree and average weighted degree are both the smallest. Therefore, survey and design companies participate in bidding activities in the market relatively infrequently.

By analyzing the network characteristics of the three types of enterprises, it is found that supervision enterprises participate in bidding activities most frequently, followed by construction enterprises, and survey and design enterprises participate in bidding activities the least frequently. In the bidding market, rational enterprises choose certain construction projects independently based on their actual capabilities. Within a certain period, their human, financial, and material resources are limited, and the number of bids they participate in should be near the market average. Therefore, the more frequent the bidding, the greater the possibility of collusion, so the possibility of collusion behavior can be ranked as supervision enterprise $>$ construction enterprise $>$ survey and design enterprise.

The modularity $Q$ of the bidder network is 0.765 , so it can be determined that the network has an obvious community structure. We used the BGLL to divide the bidder network into 181 communities and found that the various types of enterprise communities have features as small world after division. The number of the communities of the three types of enterprises is 150,20 , and 11 , respectively. The number of communities of construction enterprises accounts for the largest proportion. It can be judged that the potential quantity of collusion behaviors of enterprises is construction enterprise $>$ supervision enterprise $>$ survey and design enterprise.

It is further found that the average weighted degree of the 171 communities in the network is smaller than the average weighted degree of the network (12.205), or the number of nodes and edges of the community is small. We believe that they are less likely to participate in bidding collusion and thus are deleted. The characteristic indicators of 10 selected communities (see Table 3) show that the average node weighted degree of each community is greater than that of the entire network, and the clustering coefficients are at a relatively high level. It can be determined that these communities are more likely to participate in bidding collusion.

\subsection{The Analysis of Collusion Behavior of Typical Bidder} Communities. Since there are many internal nodes in the community divided by the BGLL, it is impossible to determine the collusive behavior of bidders in the community at the microlevel. The construction enterprise community No. 8 is selected as the typical sample for division again. According to its network characteristic value, we can see that its community structure is strong, its performance is active in the market, and it has many nodes and edges, so its collusion behavior can be analyzed at the microlevel. 
TABLE 2: Small-world eigenvalues of bidder network.

\begin{tabular}{lccccc}
\hline Project type & Number & Average degree & Average weighted degree & Clustering coefficient & Average path length \\
\hline Construction & 150 & 9.081 & 14.326 & 0.611 & 4.097 \\
Supervision & 20 & 11.631 & 26.306 & 0.635 & 3.275 \\
Survey and design & 11 & 4.596 & 7.089 & 0.741 & 3.687 \\
\hline
\end{tabular}

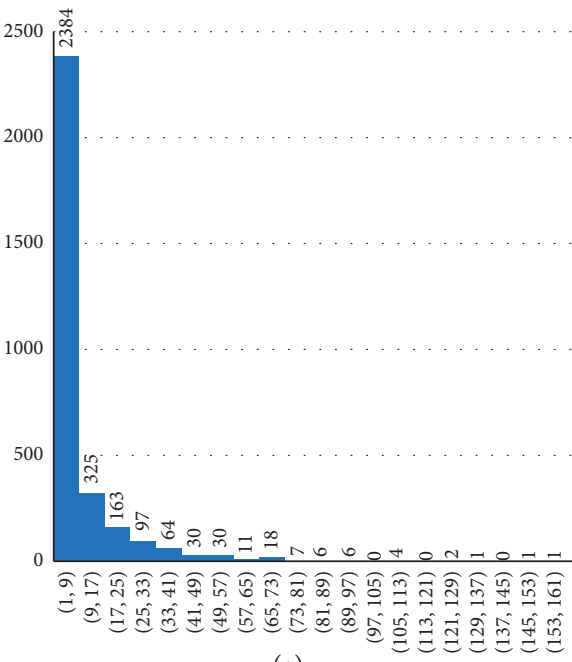

(a)

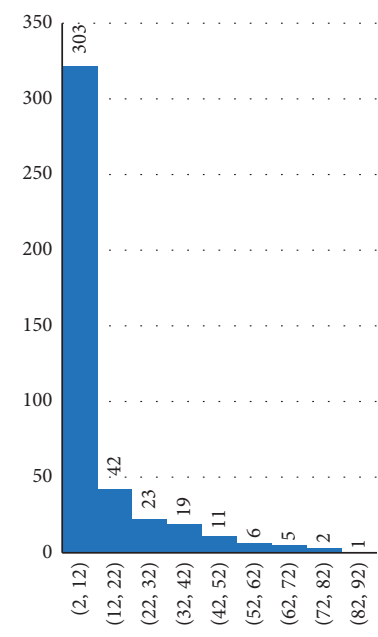

(b)

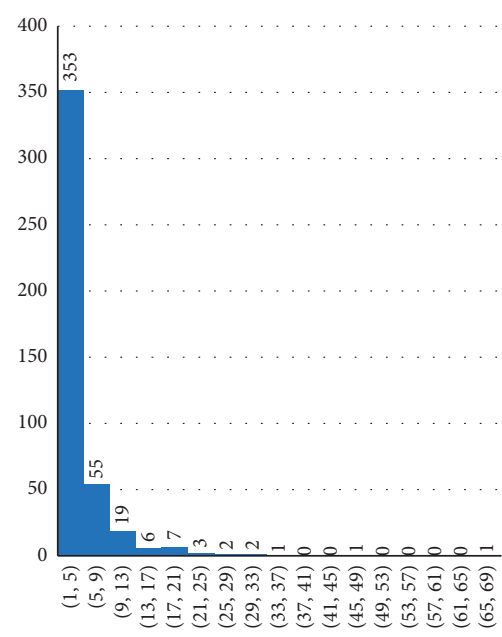

(c)

Figure 1: Degree distribution of the bidder network. (a) Construction enterprise. (b) Supervision enterprise. (c) Survey and design enterprise.

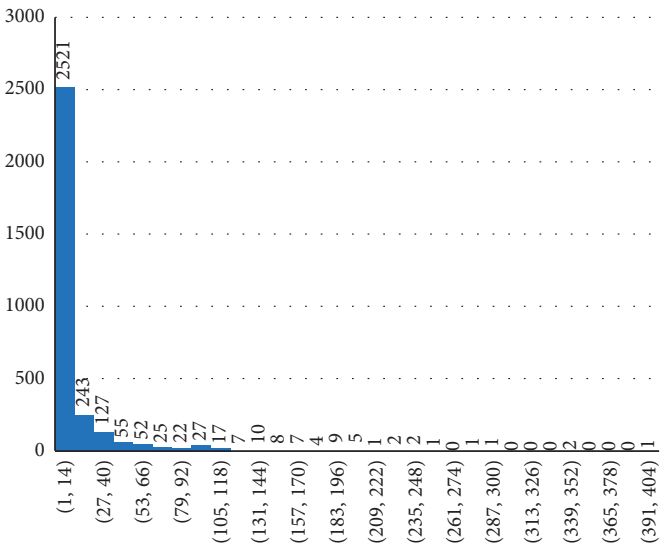

(a)

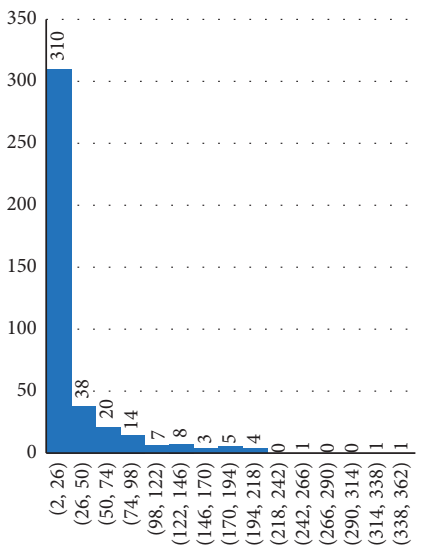

(b)

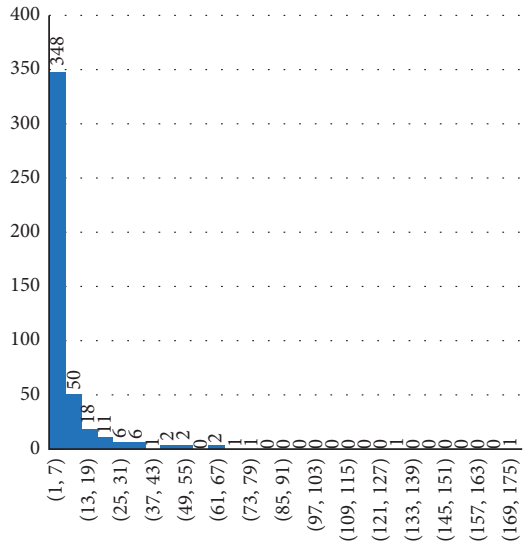

(c)

Figure 2: Weighted degree distribution of bidder network. (a) Construction enterprise. (b) Supervision enterprise. (c) Survey and design enterprise.

TABLE 3: Results of the division of network communities.

\begin{tabular}{lccccccc}
\hline Id & Node & Edge & Degree & Weighted degree & Max weighted degree & Average clustering coefficient & Project type \\
\hline 0 & 151 & 851 & 11.272 & 24.371 & 282 & 0.612 & Construction \\
6 & 48 & 115 & 4.792 & 28.208 & 403 & 0.707 & Construction \\
7 & 248 & 1296 & 10.452 & 22.685 & 208 & 0.573 & 0.669 \\
8 & 93 & 364 & 7.828 & 26.065 & 226 & 0.737 & Construction \\
10 & 224 & 1024 & 9.143 & 15.929 & 150 & 0.566 & Construction \\
48 & 493 & 2752 & 11.164 & 16.552 & 347 & 0.64 & Construction \\
118 & 43 & 81 & 3.767 & 22.837 & 193 & 0.747 & Construction \\
2 & 79 & 566 & 14.329 & 43.291 & 204 & 0.56 & Supervision \\
4 & 61 & 190 & 6.23 & 14.426 & 126 & 0.704 & Supervision \\
13 & 34 & 142 & 8.353 & 25.882 & Supervision \\
\hline
\end{tabular}


3.5. Collusion Suspicion Identification Based on Eigenvalues. The degree, weighted degree and four types of centrality indicators of No. 8 community are shown in Table 4 the top 10 nodes are selected respectively. The average degree of bidders in No. 8 community is 7.828 , and the average weighted degree is 26.065 . Among the bidders, $37.63 \%$ are above the average degree, and $23.66 \%$ are above the average degree. Judging by the bidder's centrality index, the node with greater point centrality means that the node participates in the bidding together with more nodes, and has a close relationship with other nodes in the network. For example, No. 4952 has the largest point centrality, and has participated in 68 bids with 33 bidders. The node with the largest weighted degree value is No. 2649, which has participated in 113 bids with 19 bidders. For nodes with large point centrality such as $4952,2503,2131,2562,2353,2849$, the number of bids is significantly higher than the market average. According to the centrality index determination method, these enterprises are more suspected of colluding.

The nodes with higher betweenness-centrality are in multiple network paths and have the strongest control advantage over the bidder's network, and are in the absolutely important core position in the network, such as No. 2503 and No. 4952. The betweenness centrality of No. 1746 and No. 2295 is large but closeness centrality, eigenvector centrality, and point centrality are low, indicating that these two nodes are on multiple network paths, and have control advantages in the network. But on the other hand, it has weaker influence in terms of information resources and influence.

The closeness centrality indicates the extent to which the node is not controlled by other actors. The higher the closeness centrality, the closer the node is to other nodes, the easier it is to transmit information, and the easier it is to contact other bidders, such as No. 2503, No. 2849, No. 4952 and No. 2562.

3.6. Collusion Suspicion Identification Based on Quality Evaluation of Community Division. Further division of No. 8 community is made based on the BGLL (see Figure 3). No. 8 community is further divided into 7 communities, of which 4 communities only contain 2 to 6 nodes, and the node degree and weighted degree are less than 6 . They are not active in the market, group behavior is not obvious, and the possibility of participating in collusion is extremely small, so it is eliminated, and the remaining 3 communities are $8-0$, $8-1$, and $8-2$.

The communities $8-0$ and $8-1$ have strong community structure and the communities $8-2$ is weak ones (see Table 5). All the communities are in accordance with the indicators of strong or weak community, indicating that community division is reasonable.

The core in No. 8 community is $8-0$, which not only has a strong connection among its internal nodes but also connects closely with the nodes of other two communities. The communities 8-0 and 8-1 are more structured and their nodes have stronger internal connection than the external. Therefore, they are more likely to conduct collusion in bidding. Bidder No. 2131, the core in community 8-0, which has jointly participated in biding with bidders such as No. 4952, No. 2849, No. 2503, and No. 2117 for many times, is most likely to collude. The $8-1$ community is mainly composed of bidders No. 2649, No. 4871, No. 4872, No. 4874 , and No. 4875 . They have participated in bidding together frequently and thus are more likely to commit collusion. In $8-2$ societies, the number of nodes bidding is relatively small, stable cluster structure is not found, and the possibility of collusion is relatively smaller compared with 8-0 and 8-1 communities.

CPM algorithm is further applied to detect the overlapping structure in No. 8 community. When $k=4$, the modularity $Q$ of divided network reaches the maximum. The results of community division are shown in Table 6 . The indicators of strong or weak community are shown in Table 7 . As can be seen, 8.0 community and 8.1 community conform to the indicators of weak community, showing that the division results of CPM are reasonable.

According to the division results, the bidders No. 2649 and No. 4874 appear in both communities, which indicates that they are more structured and are likely to commit collusion. In No. 8.0 community, the number of nodes whose internal intensity is greater than its external intensity is far more than that whose internal intensity is less than its external intensity. This community is more structured and is more likely for the bidders in this community to get involved in collusion.

3.7. Collusion Identification Based on the Above Two Methods. Based on the characteristic values and the community division results, the suspected degree of the 93 nodes' participation in collusion is classified according to following rules. For the nodes which have an overlapping structure and whose characteristic values exceed the average, there is a relatively high degree of participation in collusion. For the nodes which do not have an overlapping structure and a few of whose characteristic values surpass the average, there is a relatively moderate degree of participation in collusion. For the nodes which do not have an overlapping structure and whose characteristic values do not exceed the average, the degree of participation in collusion is low. The classification is shown in Table 8.

3.8. Verification of Bidder Association Behavior. In order to further verify whether the above identified bidders with a high degree of suspicion have group behaviors, an association analysis was performed on the bidding behavioral data. Since China's bidding law stipulates the requirement of bidding by at least three enterprises, the number of nodes mentioned in the preceding paragraph in the correlation analysis is to 2 . The results of the association analysis are shown in Table 9. In No. 8 community, bidder Nos. 4872, 4874, and 4875 participated in the bidding for the most times, reaching 17 times. Bidder Nos. 4872 and 4874 participated in the bidding together 37 times, of which 4875 participated 17 times, 2649 participated 10 times, and 4871 participated 8 times. Bidder Nos. 4872, 2649, and 4875 formed a group, and bidder Nos. 2649, 4871, 
TABLE 4: Degree, weighted degree and centrality indicators of each node in the 8 th society.

\begin{tabular}{lccccccccc}
\hline Node & $\begin{array}{c}\text { Weighted } \\
\text { degree }\end{array}$ & Node & $\begin{array}{c}\text { Point } \\
\text { centrality }\end{array}$ & Node & $\begin{array}{c}\text { Closeness } \\
\text { centrality }\end{array}$ & Node & $\begin{array}{c}\text { Betweenness } \\
\text { centrality }\end{array}$ & $\begin{array}{c}\text { Node } \\
\text { Eigenvector } \\
\text { centrality }\end{array}$ \\
\hline 2649 & 187 & 4952 & 35.87 & 2503 & 48.936 & 2503 & 15.707 & 2503 & 38.729 \\
2131 & 176 & 2503 & 34.783 & 2849 & 48.421 & 1746 & 12.81 & 4952 & 37.982 \\
4875 & 155 & 2131 & 28.261 & 4952 & 47.917 & 2295 & 12.031 & 2131 & 35.319 \\
4874 & 144 & 2562 & 27.174 & 2562 & 47.179 & 4952 & 11.808 & 4955 & 34.176 \\
\hline 4952 & 131 & 2353 & 26.087 & 2649 & 46.701 & 1608 & 10.428 & 2849 & 34.036 \\
4872 & 130 & 2849 & 26.087 & 2131 & 46.465 & 4871 & 10.133 & 2353 & 33.028 \\
4871 & 116 & 4955 & 26.087 & 4955 & 46.231 & 3076 & 9.417 & 1963 & 32.933 \\
2503 & 111 & 2117 & 25 & 2117 & 46 & 2230 & 8.749 & 2562 & 32.463 \\
2117 & 109 & 1963 & 25 & 2597 & 45.771 & 4874 & 8.143 & 2117 & 32.008 \\
2849 & 99 & 1264 & 22.826 & 1264 & 45.545 & 2649 & 7.522 & 1604 & 30.758 \\
\hline
\end{tabular}

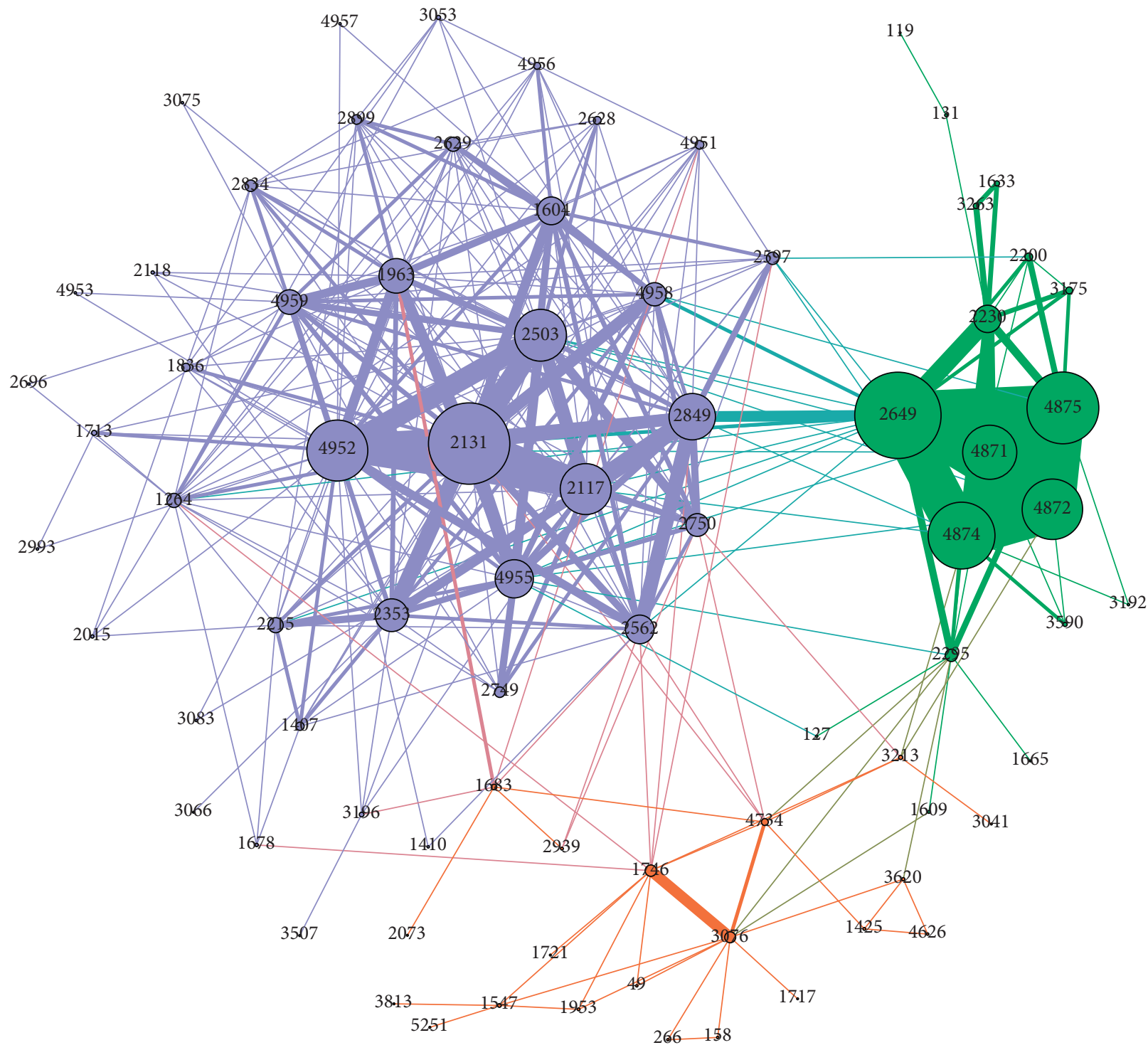

Figure 3: No. 8 community division structure in CPM algorithm.

TABLE 5: Results of strong/weak community evaluation in BGLL.

\begin{tabular}{lccc}
\hline Community & Strong/weak evaluation & Internal intensity $>$ external intensity & Internal intensity $<$ external intensity \\
\hline $8-0$ & Strong & 40 & 0 \\
$8-1$ & Strong & 18 & 0 \\
$8-2$ & Weak & 2 & 18 \\
\hline
\end{tabular}


TABLE 6: Results of the division of No. 128 in CPM algorithm.

\begin{tabular}{lcr}
\hline Community & Nodes included & Modularity \\
\hline 8.0 & $4871,4872,3175,4874,4875,2230,2295,2200,2649$ & \\
8.1 & $2562,1604,2117,2628,2503,2629,2118,4874,2834,2131,2899,4951,4952,2649,4955,4956,4958,4959,2849$, & 0.349 \\
& $2597,2215,1963,1836,1264,2353,1713,3196,2749,2750,1407$ & \\
\hline
\end{tabular}

TABLE 7: Results of strong/weak community evaluation in CPM algorithm.

\begin{tabular}{lccc}
\hline Community & Strong/weak community evaluation & Internal intensity $>$ external intensity & Internal intensity $<$ external intensity \\
\hline 8.0 & Strong & 9 & 0 \\
8.1 & Weak & 4 & 26 \\
\hline
\end{tabular}

TABLE 8: Classification of suspected degree of bidding of bidders in No. 128 community.

\begin{tabular}{lc}
\hline $\begin{array}{l}\text { Suspected } \\
\text { degree }\end{array}$ & Nodes \\
\hline $\begin{array}{l}\text { High } \\
\text { Moderate }\end{array}$ & $2649,4871,4872,4875,4874,4952,2503,2562,2849,2131,2117$ \\
& $4955,2562,1963,2353,2230,1264,4958,4959,1608,1746,1604,2750$ \\
Low & $2215,2629,2597,2834,4951,2295,3076,2899,1836,2628,2734,2749,1407,4956,1683,2200,1713,3196,3213,3053$, \\
& $3178,1547,3175,4559,5289,3620,1678,2015,2118,3590,3961,1425,2939,1953,3263,1633,131,1609,127,1410,1990$, \\
& $3083,4957,4953,2993,2696,3192,3075,49,2515,2361,266,158,3180,2978,4626,4209,1434,4026,5287,5285,3066$, \\
$1665,1721,3041,3507,2073,1717,3813,5251,119$
\end{tabular}

and 4875 also formed a group. They bid together 11 times, respectively. The bidders Nos. 4872, 4874, 4875, 2649, and 4871 formed a bidding alliance. The nodes of the alliance members held groups to participate in the bidding many times. The behavior was obviously abnormal and the suspicion of collusion was high.

\section{Discussion}

In this paper, a complex network of bidders in construction projects was established with the application of the theory and method of complex network. The possibility of bidding collusion was judged from the macro-, meso-, and microlevels by using the characteristic values and community division of the networks. According to the possibility of collusive bidding, it is proposed to divide the suspected collusive bidding into three types: red, yellow, and green. Based on a comparison of the existing literature, our discussion is as follows.

The association analysis results of No. 8 community in this paper (see Table 9) are consistent with the results of the community division (see Table 8 ), both of which identify the joint bidding behaviors. By comparing the bidders with relatively high suspected degree of collusion with the suspected companies of collusive bidding in the announcements of Shaanxi Province during 2011-2018, it is found that, in the announcements issued by the Office of Housing and Urban-Rural Development of Shaanxi Province in October 2017 [47], the companies No. 2562 and No. 2849 suspected of collusion were in the announcements due to their same pricing lock number, indicating that the level of suspected collusion identified and classified in this paper is reliable and effective. It shows that the method of bidder network community division and collusion behavior identification in construction projects is feasible.
The fact that collusion can take many forms hinders the development of a general model that can detect all types of collusive bidding behavior. This fact also led to few researchers able to tackle this problem. This paper developed a model of a social network to detect potential colluding bidders in the construction industry. This is similar to the research method of collusion bidding in Quebec's construction industry in the existing literature, "collusion can be detected by similarities in firms' interactions" [8]. At the same time, it also has similar ideas to the model proposed by Ranon Chotibhongs, and both proposed a comprehensive step-by-step method that systematically analyzes the available historical bid data and that identifies suspected cartel bidders [48]. The proposed model cannot detect collusive when collusive biddings occur; it is able to detect suspected collusion in the past and only if the analyst has access to detailed historical information. The more historical the data is, the higher the likelihood that the model will result in reliable findings.

Collusion behavior is a potential risk in the market. The current regulatory authorities in China have insufficient understanding of the early warning of bid rigging and collusion among bidders and fail to think about how to conduct effective early warning and regulation from the perspective of social risks, and most regulatory frameworks rely on whistleblowers. The most important way to effectively resolve collusion is not monitoring or punishment after collusion, but early warning. Early warning requires government departments to make judgments and take actions when a large number of decision-making factors are uncertain. The core is that measures should not to be delayed due to scientific uncertainty.

From the perspective of controlling bid rigging and collusion as early as possible and minimizing losses, the early warning of bid rigging and collusion should follow the 
TABLE 9: Results of association analysis on the behavior of bidders in No. 8 community.

\begin{tabular}{|c|c|c|c|}
\hline Latter node & Previous nodes & Number of bids participated by previous nodes & Number of joint bids \\
\hline 4875 & 4872,4874 & 37 & 17 \\
\hline 4874 & 4872,4875 & 37 & 17 \\
\hline 4872 & 4874,4875 & 31 & 17 \\
\hline 4875 & 4872,2649 & 28 & 11 \\
\hline 2649 & 4871,4875 & 24 & 11 \\
\hline 4871 & 4875,2649 & 39 & 11 \\
\hline 4872 & 4875,2649 & 39 & 11 \\
\hline 2649 & 4872,4875 & 37 & 11 \\
\hline 4875 & 4871,2649 & 31 & 11 \\
\hline 2131 & 2117,4952 & 16 & 10 \\
\hline 4952 & 2117,2131 & 27 & 10 \\
\hline 2117 & 4952,2131 & 27 & 10 \\
\hline 4874 & 4872,2649 & 28 & 10 \\
\hline 2649 & 4872,4874 & 37 & 10 \\
\hline 4872 & 4874,2649 & 36 & 10 \\
\hline 4874 & 4871,4872 & 23 & 8 \\
\hline 4872 & 4871,4874 & 20 & 8 \\
\hline 4874 & 4875,2649 & 39 & 8 \\
\hline 4871 & 4872,4874 & 37 & 8 \\
\hline 4875 & 4874,2649 & 36 & 8 \\
\hline 2649 & 4874,4875 & 31 & 8 \\
\hline 4872 & 4871,4875 & 24 & 7 \\
\hline 4875 & 4871,4872 & 23 & 7 \\
\hline 4871 & 4872,4875 & 37 & 7 \\
\hline 4871 & 4872,2649 & 28 & 6 \\
\hline 2649 & 4871,4872 & 23 & 6 \\
\hline 4871 & 2230,2649 & 15 & 6 \\
\hline 2649 & 4871,4874 & 20 & 6 \\
\hline 2649 & 2230,4871 & 11 & 6 \\
\hline 4871 & 4874,2649 & 36 & 6 \\
\hline 2230 & 4871,2649 & 31 & 6 \\
\hline 4872 & 4871,2649 & 31 & 6 \\
\hline 4874 & 4871,2649 & 31 & 6 \\
\hline 4874 & 2230,2649 & 15 & 4 \\
\hline 2503 & 4952,2131 & 27 & 4 \\
\hline 2353 & 2117,2131 & 27 & 4 \\
\hline 2649 & 2230,4874 & 6 & 4 \\
\hline 2131 & 2117,2353 & 8 & 4 \\
\hline 2117 & 2353,2131 & 13 & 4 \\
\hline 4952 & 2503,2131 & 18 & 4 \\
\hline 2131 & 2503,4952 & 17 & 4 \\
\hline 2230 & 4874,2649 & 36 & 4 \\
\hline
\end{tabular}

principle of "punishment in doubtful cases." Although the information and knowledge on which collusion warning based on is still uncertain, once the level of collusion warning is determined, the government department should take corresponding measures in a timely manner, pay attention to the behavior changes in bidding, and change or adjust the early warning level in time. Due to the concealment of the collusion, the basis for the government authorities to identify the collusion before the collusion may not be very certain. The early warning of the collusion should clearly announce the facts on which the early warning decision based on to remind the public to pay attention to it, that is, to express objectively and not make a decision lightly. The purpose is to allow the public to understand the behavior of bidders and the early warning process objectively.
The government of China promulgated the "Electronic Bidding and Bidding Law" in 2013 and introduced the electronic bidding system. In August 2015, the government of China promoted the establishment of a unified public resource electronic bidding system. The entire process of bidding realized electronic transactions. The whole process of bidding for projects that must be tendered must be carried out on the public resources trading platform. The behavioral data of bidding projects, bidders, bid evaluation experts, and other subjects are all recorded, forming a bidding database. Based on the database foundation established by the government of China, the analysis method in this paper can be popularized on the abovementioned trading platform. It is suggested that the government of China should add big data supervision platform to the system. According to the historical behavior data of each bidder, using the models and 


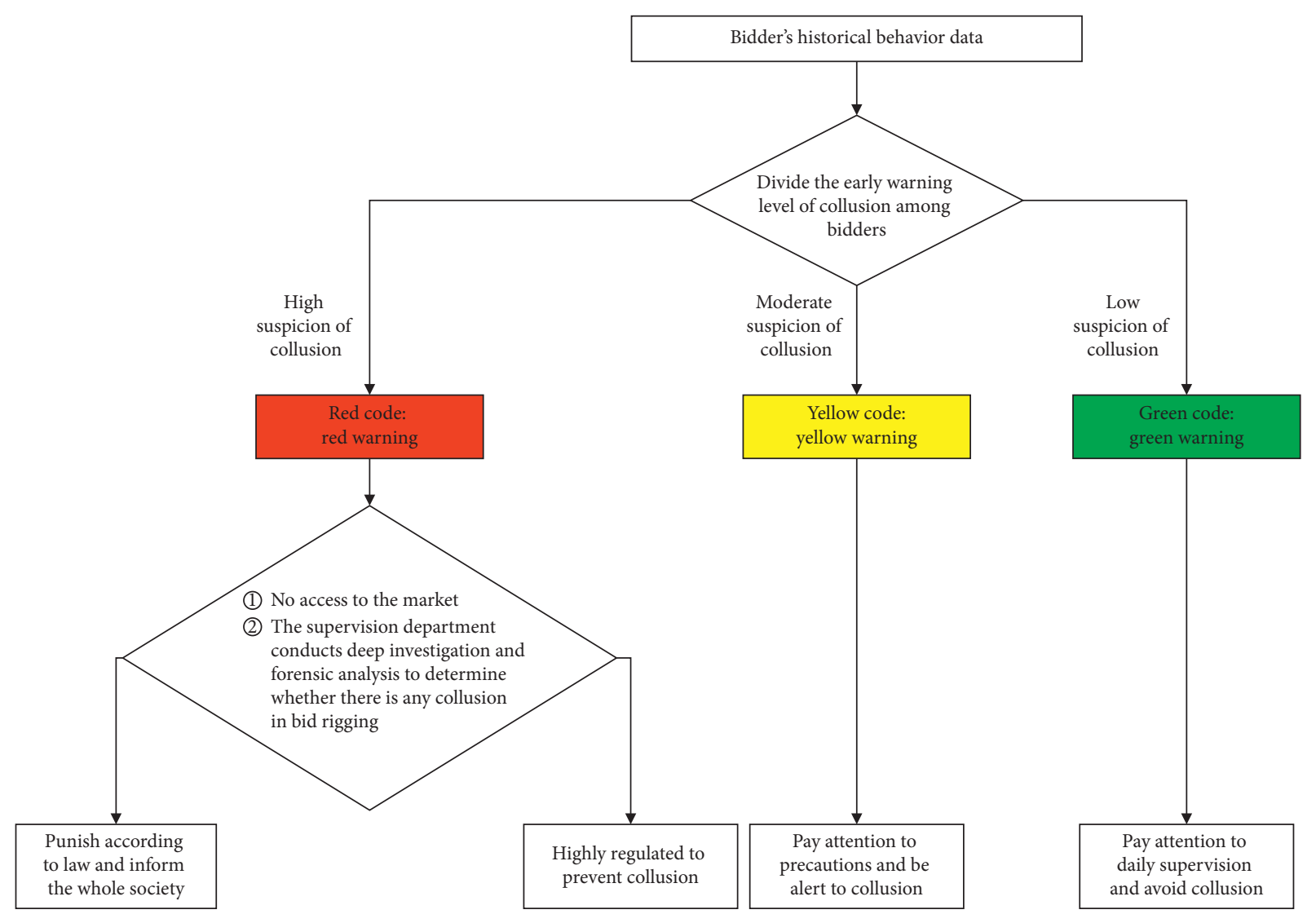

Figure 4: Three-colour warning mechanism.

methods of this article, the information of the collusion suspicion is transformed into quantifiable indicators and the corresponding analysis is performed to determine the degree of suspicion of the collusion of each bidder. According to classification of suspected degree of collusion, we proposed a red-yellow-green warning mechanism and formulated a classified response strategy for government regulatory departments to use in industry collusion supervision, as shown in Figure 4 . The classified early warning mechanism divides enterprises into extremely low collusion suspect enterprises, relatively moderate collusion suspect enterprises, and relatively high collusion suspect enterprises according to the inspected degree of collusion. For bidders $2649,4871,4872$, 4875,4874 , and 4952 with high levels of suspicion of collusion, a red warning is set; the supervisory department should carefully check their bidding activities in strict accordance with laws, regulations, rules, and policies and take measures such as reviewing joint bidding enterprises and checking bidding documents. The supervisory department shall carry out key supervision on its bidding behavior and review its historical bidding materials after bidding. If there is any bidding collusion behavior, it shall be banned from the market for a certain period. Regarding relatively moderate collusion suspect enterprises, a yellow warning is set; in addition to carefully supervising and inspecting their bidding activities in accordance with laws, regulations, rules, and policies during the bidding process, the regulatory department also needs to review the joint bidding enterprises in the bidding of these enterprises and check the bid documents carefully. Regarding extremely low collusion suspect enterprises, a green warning is set; the supervision department only needs to supervise and inspect their bidding activities in accordance with laws, regulations, rules, and policies during the bidding process, and no special supervision is required for these enterprises in the bidding. After the government department issues an early warning, it should continue to pay attention to all bidding companies and adjust the early warning level at any time according to the characteristics of the bidder's subsequent behavior.

The principle of the red, yellow, and green warning mechanism proposed in this article is similar to the health code adopted by China in the epidemic prevention and control of 2019 novel coronavirus. Health Code is divided into green, yellow, and red. The green code shows good health outing. Both yellow and red indicate home stay for the code changing into green. For the construction industry, according to the red-yellow-green warning mechanism, each enterprise has a QR code. When entering the market, the company's QR code must be shown to the regulatory authority. According to the color of the company's QR code, a differentiated and hierarchical management policy is adopted. Red code enterprises are prohibited from entering the market to participate in bidding. Yellow code enterprises need to be monitored by regulatory authorities. Green code enterprises can enjoy certain preferential policies when bidding in the market, such as exemption of bid bond. The 
red-yellow-green hierarchical management realizes ex-ante supervision, making government supervision more scientific, efficient, and accurate, and is beneficial to the efficient operation of the market.

\section{Conclusions}

Whether there is collusion in construction project, bidding has always been a problem that is difficult to discover and prove. The technology that can be used to detect suspicion of collusion is very scarce, but this research proposes an analysis method for detecting suspicion of collusion. In this study, using the theory and methods of complex networks, based on 7582 historical bidding data available in Shaanxi Province, China, a complex network containing 5293 bidders for construction projects is established with the application of the theory and method of complex network. The possibility of bidding collusion is judged from the macro-, meso-, and microlevels by using the characteristic values and community division of the networks and determines the degree of suspicion of collusion. The research findings are as follows:

(1) At the macrolevel, the bidder network of construction projects in Shaanxi Province, China, has features as small world so that the bidders may commit collusion easily. The degree of aggregation among bidders is high, the network modularity $Q$ is 0.769 , and it has an obvious community structure.

(2) At the mesolevel, there are 11 communities with high suspicion of collusion in the construction project market in Shaanxi Province, China. The communities include three types of enterprises: construction, supervision, and survey and design. Compared with construction and survey and design enterprises, the average degree value and average weighted degree value of supervision enterprises are the largest and are higher than the market average level of Shaanxi Province. They participate in the bidding most frequently and are therefore most likely to collude. The construction companies account for the largest proportion in the network, and they are of the greatest importance in the network, and there are more potential conspiring associations. Survey and design companies are the least active in the market, and the possibility of collusion is relatively small.

(3) At the microlevel, the degree of suspicion of collusion among the bidders in the typical bidder community in Shaanxi Province was identified, and three levels of suspicion of collusion were classified as high, medium, and low. Association analysis identified that Nos. 4872, 4874, 4875, 2649, and 4871 bidders formed an obvious bidding alliance, and the practical data prove that bidders No. 2562 and No. 2849 participate in the collusion. Thus, the collusion behaviors are identified effectively.

(4) A three-color early warning mechanism of "red, yellow, and green" is designed for the high, medium, and low levels of suspected collusion. For redwarning enterprises, the supervisory authority should immediately conduct evidence collection and analysis; for yellow-warning enterprises, the supervisory authority should be alert to the occurrence of collusion; green early warning companies only need to pay attention to supervision to avoid the possibility of collusion. The results of this paper can serve as reference for regulators and supervisors when establishing the early warning mechanism against collusion. Regulatory agencies can prevent collusion by using the "red-yellow- green" three-color early warning mechanism.

Of course, there are still some other research deficiencies in this paper. Since it is impossible to collect all the historical data of bidders and suspected colluding companies, this paper can only discuss the applicability of using social network methods to analyze bidding collusion behaviors. The collusion of construction projects is extremely concealed, so the results of the analysis can only identify the enterprises that may participate in the collusion, and the follow-up needs to be investigated and obtained by the regulatory department to confirm the enterprises involved in the collusion. The confidentiality level of bidding data in China is very high, and the authors cannot obtain information on all bidders of the construction project, which is not conducive to building a more accurate model. In the future, we will further expand the data sources of bidders and analyze the collusion of various relationships, such as between bidders and bidders and bidders and bidding agencies. In spite of these limitations, this study still has several useful implications, especially for the regulatory authority of the Chinese government, and this study provides helpful insight about collusive practices in the country.

Although this study focuses on the Chinese public construction projects, the methodology of this paper can be applied in other countries, especially the developing ones. Therefore, the implication of this paper can be expanded to the international scope and contribute to the global body of regulatory system for collusion behavior.

\section{Data Availability}

The data used to support the findings of this study are available from the corresponding author upon request.

\section{Conflicts of Interest}

The authors declare that there are no conflicts of interest regarding the publication of this paper.

\section{Acknowledgments}

This research was supported by the National Natural Science Foundation of China (71774132), National Key R\&D Program of China (2016YFC0401408), and Shaanxi Water Conservancy Science and Technology Project (2018SLKJ-19). 


\section{References}

[1] P. Bajari, "Comparing competition and collusion in procurement auctions: a numerical approach," Working Papers, vol. 18, pp. 187-205, 2000.

[2] A. G. Dorée, "Collusion in the Dutch construction industry: an industrial organization perspective," Building Research \& Information, vol. 32, no. 2, pp. 146-156, 2004.

[3] R. Ishii, "Collusion in repeated procurement auction: a study of a paving market in Japan," SSRN Electronic Journal, vol. 4, pp. 561-562, 2007.

[4] A. M. Lavezzi, "Economic structure and vulnerability to organised crime: evidence from Sicily," Global Crime, vol. 9, no. 3, pp. 198-220, 2008.

[5] P. Bowen, A. Akintoye, R. Pearl, and P. J. Edwards, "Ethical behaviour in the South African construction industry," Construction Management and Economics, vol. 25, no. 6, pp. 631-648, 2007.

[6] R. Signor, P. E. D. Love, A. Oliveira et al., "Public infrastructure procurement: detecting collusion in capped firstpriced auctions," Journal of Infrastructure Systems, vol. 26, 2020.

[7] A. Oke, C. Aigbavboa, and Z. Mangena, "Prevention of collusion for innovative construction," Procedia Engineering, vol. 196, pp. 491-497, 2017.

[8] C. Morselli and M. Ouellet, "Network similarity and collusion," Social Networks, vol. 55, pp. 21-30, 2018.

[9] J.-W. Zhu, L.-N. Zhou, L. Li, and W. Ali, "Decision simulation of construction project delivery system under the sustainable construction project management," Sustainability, vol. 12, 2020.

[10] Ministry of Housing and Urban-Rural Development of the People's Republic of China, Statistical Bulletin of Engineering Tendering Agencies, MOHURD, Beijing, China, 2018.

[11] M. Shan, A. P. C. Chan, Y. Le et al., "Understanding collusive practices in Chinese construction projects," Journal of Professional Issues in Engineering Education and Practice, vol. 143, 2017.

[12] O. Compte, A. Lambert-Mogiliansky, and T. Verdier, "Corruption and competition in procurement auctions," Rand Journal of Economics, vol. 36, pp. 1-15, 2005.

[13] Q. Xuan, W. Jing, and Z. Zhaoxi, "The establishment of collusion prevention system in bidding in construction market_-based on analysis of the effectiveness and feasibility of prevention measures," Engineering Sciences, vol. 10, pp. 103-112, 2013.

[14] H. C. E. "Duopoly, "Value where sellers are few," Quarterly Journal of Economics, vol. 44, pp. 63-100, 1929.

[15] J.-J. Laffont and E. Maskin, "A differential approach to dominant strategy mechanisms," Econometrica, vol. 48, no. 6, pp. 1507-1520, 1980.

[16] V. Nanduri and W. Otieno, "An application of analytics in green electricity markets with cap-and-trade programs," Engineering Management Journal, vol. 25, no. 3, pp. 74-84, 2013.

[17] D. M. Kreps, P. Milgrom, J. Roberts, and R. Wilson, "Rational cooperation in the finitely repeated prisoners' dilemma," Journal of Economic Theory, vol. 27, pp. 245-252, 1993.

[18] J.-J. Laffont and D. Martimort, "Collusion under asymmetric information,” Econometrica, vol. 65, no. 4, pp. 875-911, 1997.

[19] J. E. H. Jr, H. Kai, U. Laitenberger, and F. Smuda, "The discontent cartel member and cartel collapse: the case of the German cement cartel," International Journal of Industrial Organization, vol. 42, pp. 106-119, 2015.
[20] U. Goto and T. Iizuka, "Cartel sustainability in retail markets: evidence from a health service sector," International Journal of Industrial Organization, vol. 49, pp. 36-58, 2016.

[21] G. Aryal and M. F. Gabrielli, "Testing for collusion in asymmetric first-price auctions," International Journal of Industrial Organization, vol. 31, pp. 26-35, 2012.

[22] A. J. Björkman and S. L. Mowbray, "Putting auction theory to work: the simultaneous ascending auction," Journal of Political Economy, vol. 108, pp. 245-272, 2000.

[23] W. J. Mead, "Natural resource disposal policy-oral versus sealed bids," Natural Resources Journal, vol. 7, pp. 194-224, 1967.

[24] R. P. Mcafee and J. Mcmillan, "Analyzing the airwaves auction," Journal of Economic Perspectives, vol. 10, no. 1, pp. 159-175, 1996.

[25] S. S. Padhi and P. K. J. Mohapatra, "Detection of collusion in government procurement auctions," Journal of Purchasing and Supply Management, vol. 17, no. 4, pp. 207-221, 2011.

[26] M. Reeves-Latour and C. Morselli, "Bid-rigging networks and state-corporate crime in the construction industry," Social Networks, vol. 17, 2016.

[27] Z. Bing, L. Yun, L. Yongkui, and L. Yunbo, "Structural characteristics and attacking strategy of construction corruption network-_based on the study of dynamic network analysis," Journal of Public Management, vol. 3, pp. 33-44, 2015.

[28] Z. Bing, L. Yun, L. Yunbo, and L. Yongkui, "Corruption network diffusion mechanism under resource perspective based on multi-agent simulation," Journal of Systems \& Management, vol. 6, pp. 1091-1098, 2016.

[29] Y. Fan, "Ganxi's consequences: personal gains at social cost," Journal of Business Ethics, vol. 38, no. 4, pp. 371-380, 2002.

[30] C. Su and J. E. Littlefield, "Entering guanxi: A business ethical dilemma in mainland China?" Journal of Business Ethics, vol. 33, no. 3, pp. 199-210, 2001.

[31] A. L. Barabasi and R. Albert, "Emergence of scaling in random networks," Science, vol. 286, 1999.

[32] E. Holger, M. Lutz-Ingo, and B. Stefan, "Scale-free topology of e-mail networks," Science, vol. 66, 2002.

[33] D. J. Watts and S. H. Strogatz, "Collective dynamics of "smallworld” networks," Nature, vol. 393, no. 6684, pp. 440-442, 1998.

[34] F. J. Xie and J. Shi, "The evolution of price competition game on complex networks," Complexity, vol. 2018, 2018.

[35] M. Faloutsos, P. Faloutsos, and C. Faloutsos, "On power-law relationships of the Internet topology," ACM SIGCOMM Computer Communication Review, vol. 29, no. 4, pp. 251-262, 1999.

[36] J. Peng, Z. Lipeng, L. Chao, L. Wei, and J. Kurths, "How pricebased frequency regulation impacts stability in power grids: a complex network perspective," Complexity, vol. 2020, 2020.

[37] J. M. Hofman, A. Sharma, and D. J. Watts, "Prediction and explanation in social systems," Science, vol. 355, no. 6324, pp. 486-488, 2017.

[38] L. S. Cardona-Meza and G. Olivar-Tost, "Modeling and simulation of project management through the $\mathrm{PMBOK}{ }^{\circledR}$ standard using complex networks," Complexity, vol. 2017, 2017.

[39] E. A. Buttery and Y. H. Wong, "The development of a Guanxi framework," Marketing Intelligence \& Planning, vol. 17, no. 3, pp. 147-155, 1999.

[40] M. Girvan and M. E. J. Newman, "Community structure in social and biological networks," Proceedings of the National Academy of Sciences, vol. 99, no. 12, pp. 7821-7826, 2002. 
[41] M. E. Newman, "Fast algorithm for detecting community structure in networks," Proceedings of the National Academy of Sciences, vol. 69, 2003.

[42] C. Aaron, "Finding local community structure in networks," Physical Review E Statistical Nonlinear \& Soft Matter Physics, vol. 72, 2005.

[43] V. D. Blondel, J. L. Guillaume, R. Lambiotte, and E. Lefebvre, "Fast unfolding of communities in large networks," Journal of Statistical Mechanics, vol. 2008., pp. 155-168, 2008.

[44] M. E. J. Newman and M. Girvan, "Finding and evaluating community structure in networks," Physical Review E Statistical Nonlinear \& Soft Matter Physics, vol. 69, 2004.

[45] G. Palla, I. Derényi, I. Farkas, and T. Vicsek, "Uncovering the overlapping community structure of complex networks in nature and society," Nature, vol. 435, no. 7043, pp. 814-818, 2005.

[46] J. Wu and D. J. Watts, "Small worlds," ACM SIGMOD Record, vol. 31, no. 4, pp. 74-75, 2002.

[47] C. Shaanxi, "Provincial department of housing and urbanrural development: Xi'an. "Notice on the suspected collusion in bidding of 134 (sub) units such as Shaanxi hengde constructionengineering co.,ltd,"” 2017.

[48] R. Chotibhongs and D. Arditi, "Detection of collusive behavior," Journal of Construction Engineering and Management, vol. 138, no. 11, pp. 1251-1258, 2012. 